\title{
Comparison of range of commercial or primary care led weight reduction programmes with minimal intervention control for weight loss in obesity: Lighten Up randomised controlled trial
}

In this research paper by Kate Jolly and colleagues (BMJ 2011;343:d6500, doi:10.1136/bmj.d6500), the job title of the author Paul Aveyard was wrongly given as senior lecturer in health psychology. He is, in fact, professor of behavioural medicine.
Cite this as: BMJ 2011;343:d7243

๑ BMJ Publishing Group Ltd 2011 